\title{
CLINICAL RESEARCH ARTICLE Bubble bilevel ventilation facilitates gas exchange in anesthetized rabbits
}

Stephen C. John ${ }^{1}$, Azmath Mohammed ${ }^{2}$, Joseph T. Church ${ }^{1}$, Anna V. John ${ }^{1}$, Elena M. Perkins ${ }^{1}$, Jennifer S. McLeod ${ }^{1}$, Benjamin D. Carr ${ }^{1}$, Sue Smith ${ }^{1}$, J. Hudson Barnett ${ }^{3}$, Peter A. Gustafson ${ }^{3}$, Macdonald Dick ${ }^{1}$ and Sunil P. John ${ }^{1}$

BACKGROUND: Bubble continuous positive airway pressure is an established therapy for infants in respiratory distress. In resourcelimited settings, few treatment options exist for infants requiring further respiratory support. A bubble bilevel device has been developed to provide nonelectric, time-cycled, pressure-limited respiratory support. We compared the efficacy of bubble bilevel ventilation with conventional mechanical ventilation in sedated rabbits.

METHODS: Six adult rabbits under inhaled isoflurane general anesthesia were ventilated by alternating intervals of conventional and bubble bilevel ventilation for three 10-15-min periods. During each period, interval arterial blood gas (ABG) measurements were obtained after at least $10 \mathrm{~min}$ on the respective mode of ventilation.

RESULTS: The bubble bilevel system was able to deliver the following pressures: $20 / 7,15 / 5,12 / 5,8 / 5 \mathrm{~cm} \mathrm{H}_{2} \mathrm{O}$. The estimated differences in arterial blood gas values on bubble bilevel vs. ventilator were as follows (normalized values): $\mathrm{pH}^{7.41} \mathrm{vs} .7 .40, \mathrm{pCO}_{2}$ 37.7 vs. $40, \mathrm{pO}_{2} 97.6$ vs. 80 . In addition, the bubble bilevel ventilation delivered consistent pressure waveforms without interruption for over $60 \mathrm{~min}$ on two rabbits.

CONCLUSION: This study demonstrates promising in vivo results on the efficacy of a novel bubble bilevel device, which may prove useful for infants in respiratory distress.

Pediatric Research (2021) 89:622-627; https://doi.org/10.1038/s41390-020-0928-0

\section{IMPACT:}

- Given the lack of personnel, funds or infrastructure to provide neonatal mechanical ventilation in resource-limited settings, additional low-cost, low-tech treatments are necessary to save infant lives.

- Bubble bilevel ventilation reliably delivers two levels of airway pressure to anesthetized rabbits resulting in normalization of blood gases comparable to those achieved on a traditional ventilator.

- If proven effective, simple technologies like this device have the potential to significantly impact neonatal mortality due to respiratory distress globally.

\section{INTRODUCTION}

The World Health Organization estimates that over 1 million neonates die each year due to respiratory causes. ${ }^{1}$ Infants with conditions such as apnea, respiratory distress syndrome, and pneumonia constitute a large group who are at risk for respiratory failure. These infants, as well as those recovering from surgery, often require noninvasive respiratory support. Lack of access to supportive respiratory therapies contributes to the large infant mortality from respiratory failure. While intensive care and mechanical ventilation have been widely adopted in developed countries, these solutions remain out of reach in many resourcelimited settings. ${ }^{2}$

Bubble continuous positive airway pressure (bubble CPAP) is a safe and effective therapy for infants with mild respiratory distress. ${ }^{3}$ It is a low-cost, simple-to-use technology, which has been widely adopted throughout the world. ${ }^{2}$ Although many infants improve with initiation of bubble CPAP, it is not effective in the treatment of some infants with moderate or severe respiratory distress. Bilevel positive airway pressure (bilevel PAP) and nasal intermittent positive pressure ventilation (NIPPV) are wellestablished noninvasive treatments for infants with more severe respiratory distress. ${ }^{4}$ Currently these treatments require mechanical ventilators or dedicated bilevel PAP machines, neither of which are readily available in resource-limited settings. ${ }^{3}$

A bubble bilevel ventilation device, NeoVent, has been developed that delivers time-cycled, pressure-limited ventilation. The device utilizes a variable buoyancy float, which captures the bubbles from bubble CPAP. This energy is used to change the effective water column depth and cycle between a low- and highpressure level. In comparison with bubble CPAP, the device has minimal increase in user complexity and estimated cost. Promising results of bench testing in an inanimate model have been previously reported. ${ }^{5,6}$ Here we report the results of in vivo testing of bubble bilevel ventilation using this device in deeply anesthetized rabbits. Our aim was to test the ability of bubble bilevel ventilation to provide pressures comparable to a

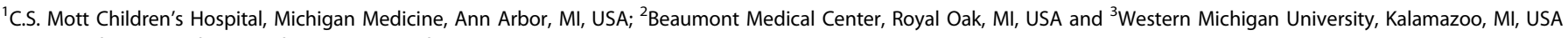
Correspondence: Stephen C. John (scjres@gmail.com) 
conventional mechanical ventilator with appropriate gas exchange as demonstrated by arterial blood gas (ABG) analysis.

\section{METHODS}

Bilevel PAP modification

The NeoVent consists of an oscillatory valve, which uses the bubbles of bubble CPAP to power cycling between two levels of pressure. In the low-pressure state, bubbles emerge from proximal holes in the submerged expiratory limb of tubing. As in the case of bubble CPAP, this submerged depth of bubbling determines the pressure. A float collects these bubbles, becomes buoyant and rises. In the process, the float pulls up an attached sleeve, which occludes the proximal holes. The air column is forced to travel deeper in the water, to a depth proportional to the value set by a pressure regulator valve external to the water. The float then vents the bubbles, becomes heavy and sinks. The attached sleeve slides down, opening the proximal holes; bubbling from the proximal holes resumes and the pressure decreases to the lower level. This process cyclically repeats, resulting in a controllable bilevel pressure waveform. The levels of pressure are set hydrostatically and can be visually verified by the depth of the water column, similar to bubble CPAP. Specifically, filling the container with additional water increases the low-pressure level while removing water decreases the low-pressure level. The pressure regulator valve can be adjusted to determine the depth to which the air column travels, setting the high level of pressure. (Both low- and high-pressure levels can be adjusted without interrupting device function or introducing contaminants to the water.) The rate of cycling between both pressures is set by the airflow rate; at a high airflow rate, the float becomes buoyant more quickly, decreasing the overall cycling time. Note that the high-pressure level, lowpressure level and cycling rate can all be set independently (Fig. 1).

\section{Surgical preparation}

The experiments were conducted under the University of Michigan Institutional Animal Care and Use Committee (IACUC) protocol 6897 and approved by the University of Michigan Laboratory Animal Welfare committee. Animals were treated according to the Guide for Care and Use of Laboratory Animals 8th edition (US National Institutes of Health publication No. 85-23, National Academy Press, Washington D.C., revised 1996).

Six adult white New Zealand rabbits with mean body weight $3.0 \mathrm{~kg}$ (S. Dev. 0.3) constituted the animal study group for bubble bilevel ventilation. This model was selected to approximate the weight and lung size of an infant. Rabbits were anesthetized by intramuscular injection of ketamine $30 \mathrm{mg} / \mathrm{kg}$ and xylazine $5 \mathrm{mg} /$ $\mathrm{kg}$. Inhalational isoflurane gas at $0.5-3 \%$ (initially via face mask and subsequently via endotracheal tube) was used to ensure adequate anesthesia and decrease spontaneous respirations. The marginal ear vein was cannulated to administer IV fluid and medications. The animals were placed in the supine position and underwent tracheotomy just below the cricoid cartilage. An uncuffed endotracheal tube of $3.0 \mathrm{~mm}$ inner diameter was inserted in the trachea and secured with a tie. The carotid artery was cannulated to obtain samples for blood gas analysis. A catheter with normal saline containing heparin was introduced into the carotid artery for continuous arterial blood pressure measurement.

\section{Ventilation procedure}

General anesthesia was maintained with inhaled isoflurane connected in line with the inspiratory limb of the respiratory circuit. During the experiment, airway pressure was monitored continuously at the endotracheal tube $y$-connector. The pressure waveforms were recorded with custom-developed computer data acquisition system with a Honeywell162PC01D pressure transducer for subsequent analysis. The pressure measurement system

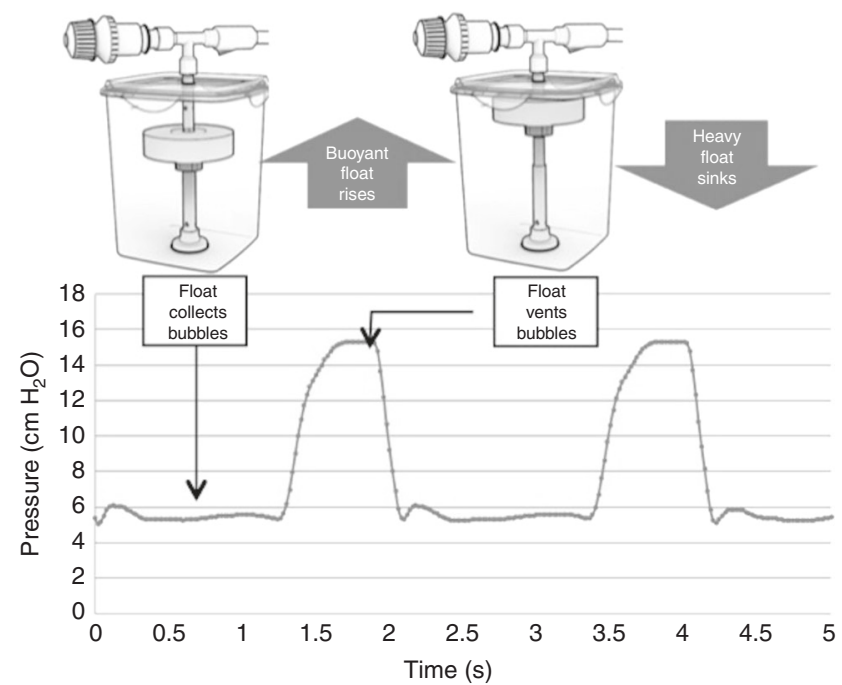

Fig. 1 NeoVent functional diagram.

was calibrated with a water column manometer prior to the start of the study.

A case control study design was utilized in which each rabbit could serve as its own control. After initial stabilization on a conventional ventilator, bubble bilevel ventilation with NeoVent was alternated with conventional ventilation in a crossover design for three periods lasting $10-15 \mathrm{~min}$ (total time $60-90 \mathrm{~min}$ ). This duration of $10-15$ min per treatment (with an ABG recorded at $10 \mathrm{~min}$ on each treatment) was selected to reduce the carry-over of $\mathrm{pH}, \mathrm{pO}_{2}, \mathrm{pCO}_{2}$ and $\mathrm{SaO}_{2}$ values from the previous treatment. Two of the rabbits underwent two periods of NeoVent/mechanical ventilation instead of three periods. In lieu of the third period, these rabbits continued on NeoVent for $60 \mathrm{~min}$ to assess the ability of NeoVent to reliably ventilate animals over a longer period of time (Fig. 2).

The ventilation circuits are depicted in Fig. 3. To switch between circuits, the inspiratory limb of the circuit was removed from the mechanical ventilator and connected to a compressed air source, while the expiratory limb of the circuit was connected to the NeoVent. Continuous pulse oximetry and end-tidal capnography were used throughout the experiment to instantaneously monitor oxygenation and carbon dioxide clearance. During each period an ABG was obtained at least 10 min into each therapy interval.

Targeting pressure levels of 20/7, $15 / 5$ and $8 / 5 \mathrm{~cm} \mathrm{H}_{2} \mathrm{O}$ with NeoVent. To test the ability of NeoVent to deliver different pressure gradients, a range of pressures was selected for rabbits 1, 2, and 3. A veterinary anesthetic ventilator (ADS 2000; Engler: Hialeah, FL) was alternated with the NeoVent. The ventilator and NeoVent pressure settings were systematically varied from 20/7 to $15 / 5$ to $8 / 5 \mathrm{~cm} \mathrm{H}_{2} \mathrm{O}$ for periods 1,2 , and 3, respectively. The cycling rate was set to approximately 30 cycles/min.

Ability of NeoVent to "rescue" a rabbit in respiratory failure. Subsequently, rabbits 1,2 , and 3 underwent a brief period with blow-by air until the oxygen saturation decreased below $70 \%$. This was done to confirm depth of sedation and lack of spontaneous respiratory drive. Support with the NeoVent was reinitiated when oxygen saturation decreased below $70 \%$.

Targeting a tidal volume of $7 \mathrm{ml} / \mathrm{kg}$ with NeoVent. Rabbits 4, 5, and 6 underwent alternating ventilation with the NeoVent and an Avea ventilator (Carefusion: Yorba Linda, CA). The Avea ventilator's pneumotachometer was used to measure tidal volumes delivered by the NeoVent. The $\mathrm{P}$ high and $\mathrm{P}$ low were adjusted to deliver 


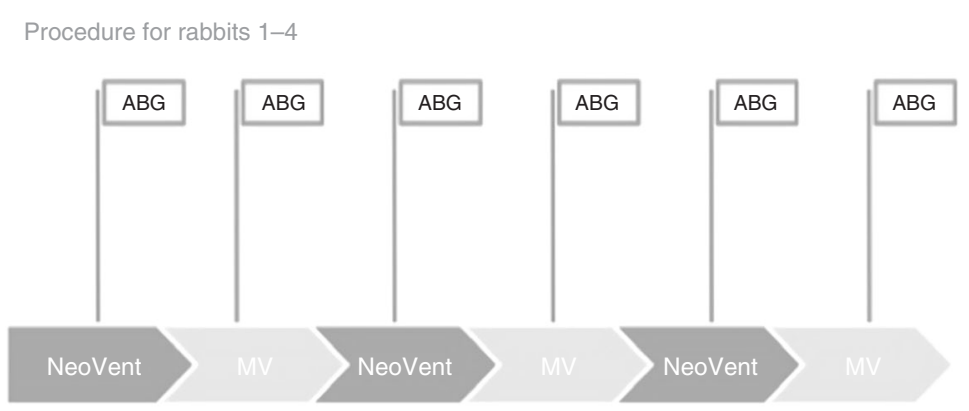

Procedure for rabbits 5-6

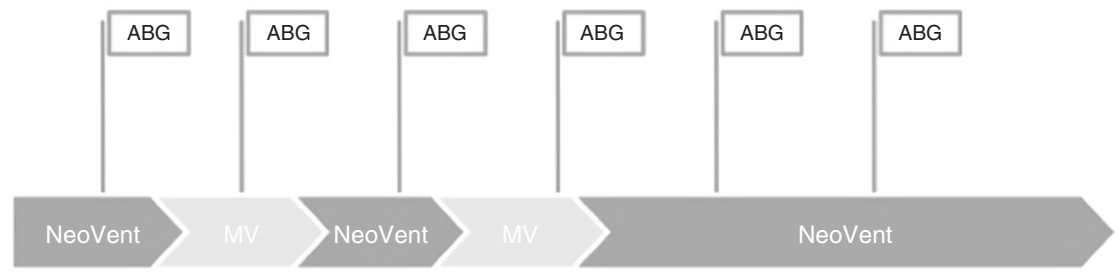

Fig. 2 Experimental procedure.
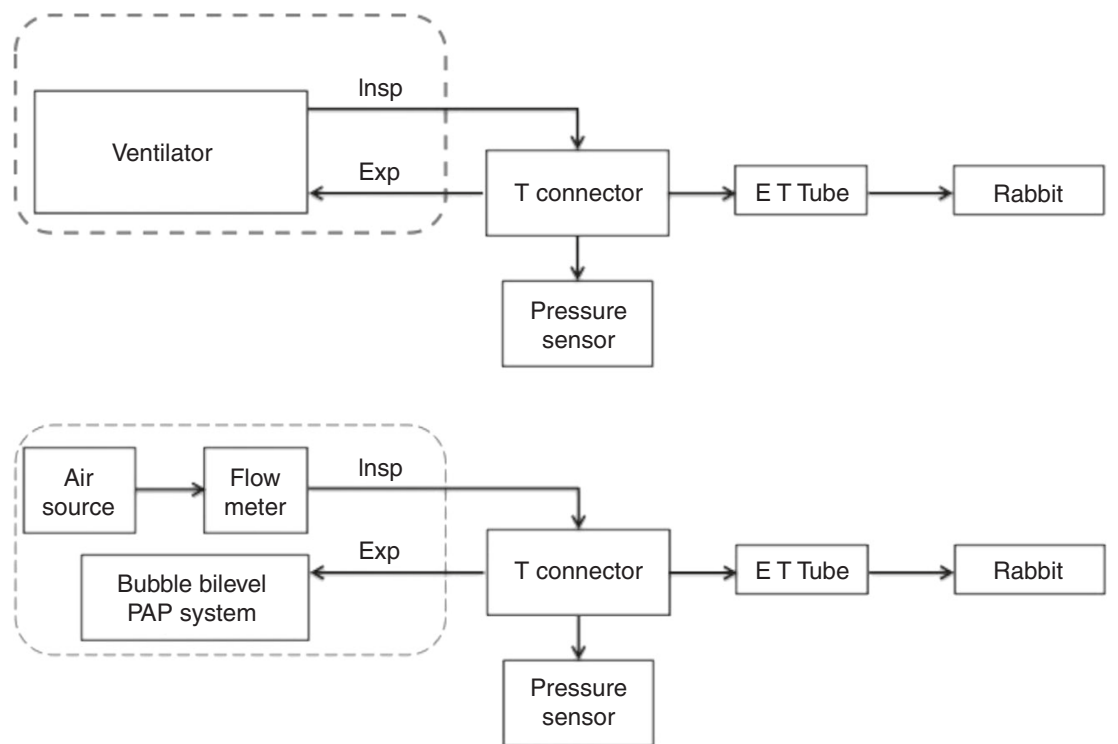

Fig. 3 Respiratory circuits for ventilator and bubble bilevel ventilation.

tidal volumes of approximately $7 \mathrm{ml} / \mathrm{kg}$, which corresponded to pressures of approximately $12 / 5 \mathrm{~cm} \mathrm{H}_{2} \mathrm{O}$. The NeoVent cycling rate was set around 60 cycles/min for these three rabbits.

Ability of NeoVent to ventilate for 60 min continuously. In rabbits 5 and 6, after initially alternating between bubble bilevel PAP and the ventilator for two periods per method of ventilation, rabbits were ventilated exclusively using the NeoVent for over 60 min with ABGs collected at 30-min intervals to assess device reliability.

Statistical methods

Statistical analyses were completed using SAS/STAT (Version 9.4, SAS Institute, Cary, NC). For each quantitative response variable, a generalized linear mixed model (GLMM) was fit to the data, using the fixed effects of treatment (NeoVent or Vent), time, low pressure, and pressure gradient. Random effects included rabbit and treatment within rabbit. A $90 \%$ confidence level was used for each GLMM fit to these data.
To accommodate a two-one-sided $t$ test (TOST) procedure and assess NeoVent vs. Vent equivalence, the data were additionally log transformed and analyzed using the same GLMM described above. This allowed us to compare the percent difference between NeoVent and Vent while inverting the underlying statistical hypotheses, thus providing an appropriate penalty considering the under-powered nature of this study. For each NeoVent vs. Vent comparison, an $80 \%$ confidence interval for the estimated percent difference was calculated, corresponding to an overall $90 \%$ TOST confidence level.

\section{RESULTS}

Pressure delivered by bubble bilevel ventilation NeoVent was able to deliver the following pressures consistently: $20 / 7,15 / 5,12 / 5$, and $8 / 5 \mathrm{~cm} \mathrm{H}_{2} \mathrm{O}$. The set cycling rate was varied from $\sim 30$ to $\sim 60$ cycles/min by changing the flow of compressed air $/ \mathrm{O}_{2}$ in the circuit. Representative pressure waveforms over time 


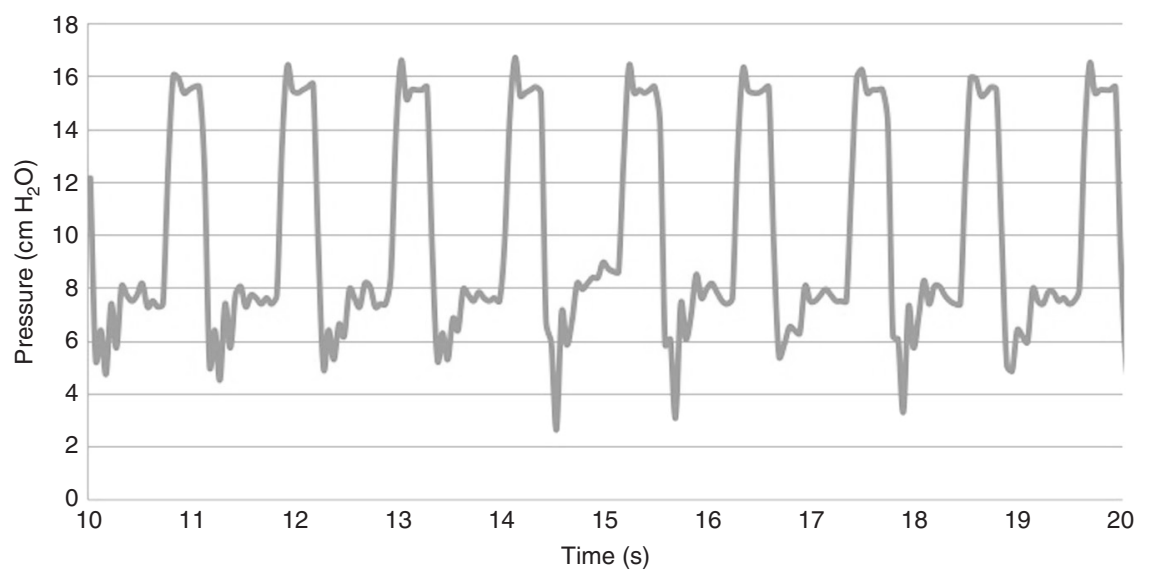

Fig. 4 Bubble bilevel pressure waveform over time.

on bubble bilevel and are shown in Fig. 4. NeoVent delivered airway pressures similar to those of the mechanical ventilator throughout the study.

Tidal volumes delivered by bubble bilevel ventilation Lung expansion was confirmed with the clinical picture of vitals, chest rise and auscultation. Tidal volumes were measured using a hot-wire pneumo-tachometer at the endotracheal tube Y-connector. The mean (standard deviation) inspiratory tidal volume on bubble bilevel ventilation was 7.7 (1.7) $\mathrm{ml} / \mathrm{kg}$ for the rabbit that received $60+$ min of continuous NeoVent treatment. In comparison, the mean (standard deviation) inspiratory tidal volume while on the Avea ventilator was $6.8(0.7) \mathrm{ml} / \mathrm{kg}$. Flow volume-loops on NeoVent were similar to those on conventional mechanical ventilation.

Arterial blood gas measurements and pulse-oximetry Arterial blood gas values were within the acceptable ranges.

Table 1 displays the estimated difference in ABG values on bubble bilevel vs. ventilator, with ventilator values normalized to $7.40,40,80,24\left(\mathrm{pH}, \mathrm{pCO}_{2}, \mathrm{pO}_{2}, \mathrm{HCO}_{3}\right)$.

Figure 5 shows this information in a different format: the estimated \% difference $(80 \% \mathrm{Cl})$ for $A B G$ values of $\mathrm{pH}, \mathrm{pCO}_{2}, \mathrm{pO}_{2}$, $\mathrm{HCO}_{3}$ are displayed for bubble bilevel in comparison with the conventional vent.

Continuous bubble bilevel operation

In rabbits 5 and 6 , during prolonged continuous use of the bubble bilevel system for over $60 \mathrm{~min}$, the device cycled continuously without interruption. Mean (standard deviation) of $A B G$ values during this period were as follows: $\mathrm{pH} 7.4$ (0.03), $\mathrm{pCO}_{2} 46$ (6.8) and $\mathrm{pO}_{2} 96$ (21).

Rescue bubble bilevel ventilation

When the first three anesthetized rabbits received blow-by air only, their oxygen saturation rapidly decreased to less than $70 \%$. The oxygen saturation rapidly returned to greater than $96 \%$ with rescue by bubble bilevel ventilation.

\section{DISCUSSION}

This initial in vivo study in anesthetized rabbits demonstrates that NeoVent provides bilevel positive pressure ventilation similar to that of a conventional mechanical ventilator. There were small and clinically insignificant estimated percent differences of $\mathrm{ABG} \mathrm{HCO}_{3}, \mathrm{pCO}_{2}$ and $\mathrm{pH}$ values for the bubble bilevel system as compared to the conventional ventilator. It may be that the slight increase in $\mathrm{CO}_{2}$ clearance with the NeoVent reflects enhanced gas exchange due to the high frequency
Table 1. Estimated difference in arterial blood gas values on bubble bilevel vs. ventilator (normalized values).

\begin{tabular}{llll}
\hline & Ventilator & Bubble bilevel & NeoVent $80 \% \mathrm{Cl}$ \\
\hline $\mathrm{pH}$ & 7.40 & 7.41 & $7.38-7.45$ \\
$\mathrm{pCO}_{2}$ & 40 & 37.7 & $33.7-42.2$ \\
$\mathrm{pO}_{2}$ & 80 & $97.6^{\mathrm{a}}$ & $81.0-117.1$ \\
$\mathrm{HCO}_{3}$ & 24 & 23.7 & $22.2-25.2$ \\
\hline
\end{tabular}

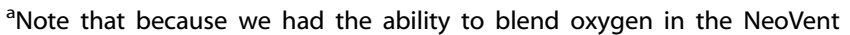
circuit but not the ventilator circuit, the comparison of arterial oxygen levels may not be as relevant

oscillations in pressure from the bubbling. A similar effect has been noted in bubble CPAP in comparison with ventilator-based CPAP. ${ }^{7}$ Of note, the improvement of $\mathrm{pO}_{2}$ on the bubble bilevel system was likely due to the blended mixture of oxygen and air, which powered the system.

The tidal volumes were on average within $0.9 \mathrm{ml} / \mathrm{kg}$ of those delivered by a conventional ventilator. The increased tidal volumes with NeoVent are consistent with the above-noted increased clearance of $\mathrm{CO}_{2}$; again, this may be due to enhanced gas exchange due to the high frequency oscillations in pressure due to bubbling. The pressure waveforms appear similar to those delivered by a conventional ventilator. The device functioned continuously for over $90 \mathrm{~min}$ without interruption of cycling.

Most significantly, bubble bilevel ventilation was able to support effective gas exchange in vivo with normal oxygen saturation and $A B G$ values. All six animals demonstrated normal ABGs while supported by bubble bilevel ventilation. Bubble bilevel support was able to rapidly restore the oxygen saturation following desaturation while on blow-by room air.

Current treatments for respiratory distress in infants Continuous positive airway pressure (CPAP) has been shown to be safe and effective for neonates in respiratory distress. ${ }^{2,8}$ In a preterm lamb model, bubble CPAP was shown to enhance lung volume and improve gas exchange in comparison with ventilator CPAP. ${ }^{9}$ Among neonates in respiratory distress, bubble CPAP has led to greater success and shorter hospital length of stay than ventilator CPAP. ${ }^{10}$ The simplicity and low cost of bubble CPAP ${ }^{11}$ have facilitated its implementation in hospitals around the world over the last 20 years.

Infants with a more severe respiratory distress require additional treatment such as bilevel PAP ${ }^{12}$ or NIPPV., ${ }^{43}$ In a randomized control trial, bilevel PAP led to reduced hospital stay and 


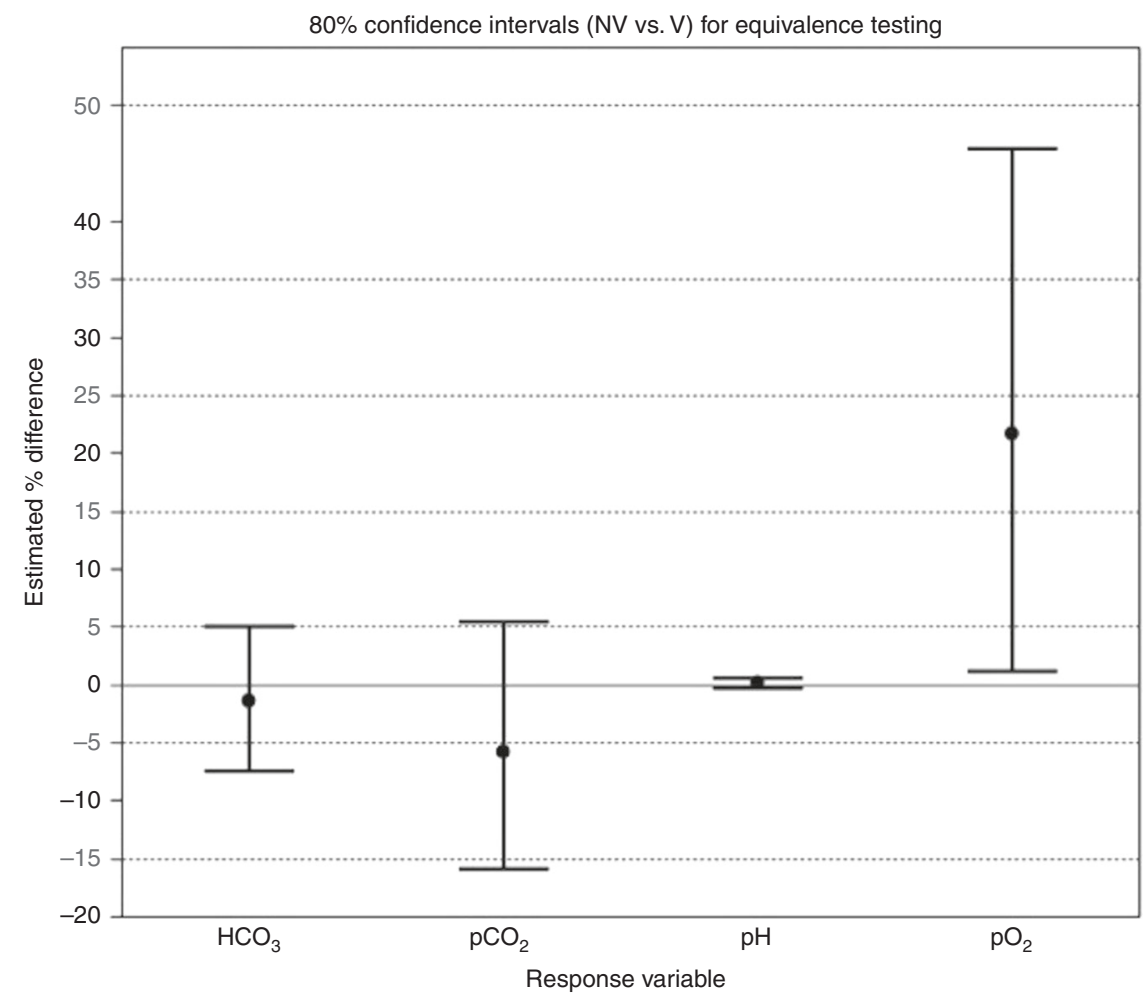

Fig. 5 Estimated \% difference $(80 \% \mathrm{Cl})$ for $\mathrm{HCO}_{3}, \mathrm{pCO}_{2}, \mathrm{pH}$ and $\mathrm{pO}_{2}$ on bubble bilevel vs. conventional vent.

decreased oxygen need in comparison with CPAP among preterm infants with respiratory distress syndrome. In a crossover study, infants demonstrated superior gas exchange when on bilevel PAP in comparison with CPAP. ${ }^{14}$ While subsequent trials have not confirmed this benefit, they have had relatively short crossover periods of study. ${ }^{15}$ NIPPV has been shown to reduce treatment failure leading to intubation and mechanical ventilation in comparison to CPAP. ${ }^{16-20}$ In a single-center randomized control trial, the time on treatment and oxygen requirements were both decreased in a group treated with NIPPV in comparison with bubble CPAP. ${ }^{21}$ Note that both synchronized and asynchronized NIPPV have been shown to be beneficial; ${ }^{4}$ clinical experience suggests that if the ventilator does not synchronize with the infant, the infant may synchronize with the ventilator.

Challenges of resource-limited settings

Because there are currently few simple and low-cost mechanisms to deliver bilevel PAP, NIPPV or mechanical ventilation, many hospitals in resource-limited settings are only able to offer bubble CPAP; infants who do not respond to bubble CPAP die for lack of additional respiratory support. There is an urgent need for additional affordable and simple-to-use ventilation technologies.

Our data indicate that a bubble bilevel PAP system can provide respiratory support comparable to conventional mechanical ventilation in terms of pressure and volume delivery, and supporting adequate gas exchange. Because the nonelectric design preserves the simplicity of bubble CPAP, it may be of great utility in resource-limited settings in infants for whom bubble CPAP is ineffective.

\section{Limitations}

The current study has a number of limitations. This proof-ofconcept work is limited to an analysis demonstrating the consistency of airway pressures delivered and normalization of $A B G s$. Concern for sufficient washout period was mitigated by the observation that, clinically, the animals appeared to have stabilized (e.g. per vital signs) well before the 15-min time period was complete. In addition, other animal studies of simple ventilation devices utilize a crossover design with treatment durations of $10-15$ min. $^{22}$

Second, the depth of anesthesia varied with the animals. At higher concentrations of isoflurane, the animals became hypotensive. The experiment required that the animals be sufficiently anesthetized to abolish spontaneous respiration, but not so deep as to precipitate hemodynamic instability. A sedated adult rabbit may not be representative of an infant in respiratory distress (e.g. different values of compliance and resistance), but does provide a good model of physiology in a comparably sized animal. Further experiments could be undertaken to investigate the delivery of pressures and volumes in diseased lungs.

Third, the NeoVent is designed to deliver bilevel PAP noninvasively via nasal cannula to infants in respiratory distress. However, a suitable interface for occlusive application of nasal cannula was not available in the rabbit model. Consequently, treatment was delivered through an endotracheal tube and tracheostomy. Tracheostomy and arterial cannulation procedure are associated with their own complications. Prior to placement on NeoVent or mechanical ventilation, three rabbits died of hemodynamic instability after tracheostomy and were not included in the study group. Future experiments could investigate mechanisms to deliver the same support noninvasively in an animal model.

The above limitations non-withstanding, these data are the first to be published with this device in an in vivo model and are critical to device development.

\section{CONCLUSION}

Given the lack of personnel, funds or infrastructure to provide neonatal mechanical ventilation in resource-limited settings, additional low-cost, low-tech treatments are necessary to save infant lives. This study demonstrates that bubble bilevel ventilation reliably delivers two levels of airway pressure to anesthetized 
rabbits resulting in normalization of blood gases. These promising preclinical results represent the first steps in validation of device function. If proven effective, simple, low-tech technologies like this device have the potential to significantly impact on global neonatal mortality due to respiratory distress.

\section{ACKNOWLEDGEMENTS}

All phases of this study were supported by internal funding from the Division of Pediatric Cardiology, Department of Pediatrics, C.S. Mott's Children's Hospital, Michigan Medicine, University of Michigan.

\section{AUTHOR CONTRIBUTIONS}

S.C.J. designed the device, conceptualized and designed the study, assisted with the experiments, drafted the manuscript and approved the final manuscript as submitted. A.M. conceptualized and designed the study, conducted the experiments, reviewed the manuscript and approved the final manuscript as submitted. J.T.C. conceptualized the study, assisted with the experiments, reviewed the manuscript and approved the final manuscript as submitted. A.V.J. designed the device, assisted with the experiments, reviewed the manuscript and approved the final manuscript as submitted. E.M.P., J.S.M., B.D.C., S.S. and J.H.B. assisted with the experiments, reviewed the manuscript and approved the final manuscript as submitted. P.A.G. designed the pressure monitoring system, assisted with the experiments, completed the statistical analysis, reviewed the manuscript and approved the final manuscript as submitted. M.D. conceptualized and reviewed the study, reviewed the manuscript and approved the final manuscript as submitted. S.P.J. conceptualized and designed the study, conducted the experiments, drafted the manuscript and approved the final manuscript as submitted.

\section{ADDITIONAL INFORMATION}

Competing interests: P.A.G., J.H.B., S.C.J. and A.V.J. have filed patent protection for bubble bilevel positive airway pressure technologies. P.A.G., J.H.B., A.V.J. and S.C.J. have worked for Advanced Innovative Medical Technologies, LLC. The other authors have indicated they have no potential conflicts of interest to disclose.

Publisher's note Springer Nature remains neutral with regard to jurisdictional claims in published maps and institutional affiliations.

\section{REFERENCES}

1. Liu, L. et al. Global, regional, and national causes of under-5 mortality in 2000-15: an updated systematic analysis with implications for the Sustainable Development Goals. Lancet 388, 3027-3035 (2016).

2. Duke, T. CPAP: a guide for clinicians in developing countries. Paediatr. Int. Child Health 34, 3-11 (2014).

3. Martin, S., Duke, T. \& Davis, P. Efficacy and safety of bubble CPAP in neonatal care in low and middle income countries: a systematic review. Arch. Dis. Child. Fetal Neonatal Ed. 99, F495-F504 (2014).

4. Bhandari, V. Nasal intermittent positive pressure ventilation in the newborn: review of literature and evidence-based guidelines. J. Perinatol. 30, 505-512 (2010).
5. John, S. C. et al. Development and testing of a bubble bi-level positive airway pressure system. Respir. Care 62, 1131-1136 (2017).

6. John, S. C. et al. Bench testing of a bubble noninvasive ventilation device in an infant lung simulator. Respir. Care (2020). in press.

7. Lee, K. S., Dunn, M. S., Fenwick, M. \& Shennan, A. T. A comparison of underwater bubble continuous positive airway pressure with ventilator-derived continuous positive airway pressure in premature neonates ready for extubation. Neonatology 73, 69-75 (1998).

8. Urs, P. S., Khan, F. \& Maiya, P. P. Bubble CPAP—a primary respiratory support for respiratory distress syndrome in newborns. Indian Pediatr. 46, 409-411 (2009).

9. Pillow, J. J. et al. Bubble continuous positive airway pressure enhances lung volume and gas exchange in preterm lambs. Am. J. Respir. Crit. Care Med. 176, 63-69 (2007).

10. Tagare, A., Kadam, S., Vaidya, U., Pandit, A. \& Patole, S. Bubble CPAP versus ventilator CPAP in preterm neonates with early onset respiratory distress-a randomized controlled trial. J. Trop. Pediatr. 59, 113-119 (2013).

11. Chen, A. et al. Cost-effectiveness analysis of a low-cost bubble CPAP device in providing ventilatory support for neonates in Malawi-a preliminary report. BMC Pediatr. 14, 288 (2014).

12. Lista, G. et al. Nasal continuous positive airway pressure (CPAP) versus bi-level nasal CPAP in preterm babies with respiratory distress syndrome: a randomised control trial. Arch. Dis. Child. Fetal Neonatal Ed. 95, F85-F89 (2010).

13. Davis, P. G., Morley, C. J. \& Owen, L. S. Non-invasive respiratory support of preterm neonates with respiratory distress: continuous positive airway pressure and nasal intermittent positive pressure ventilation. Semin. Fetal Neonatal Med. 14, 14-20 (2009).

14. Migliori, C., Motta, M., Angeli, A. \& Chirico, G. Nasal bilevel vs. continuous positive airway pressure in preterm infants. Pediatr. Pulmonol. 40, 426-430 (2005).

15. Lampland, A. L., Plumm, B., Worwa, C., Meyers, P. \& Mammel, M. C. Bi-level CPAP does not improve gas exchange when compared with conventional CPAP for the treatment of neonates recovering from respiratory distress syndrome. Arch. Dis. Child. Fetal Neonatal Ed. 100, F31-F34 (2015).

16. Mahmoud, R. A., Roehr, C. C. \& Schmalisch, G. Current methods of non-invasive ventilatory support for neonates. Paediatr. Respir. Rev. 12, 196-205 (2011).

17. Tang, S., Zhao, J., Shen, J., Hu, Z. \& Shi, Y. Nasal intermittent positive pressure ventilation versus nasal continuous positive airway pressure in neonates: a systematic review and meta-analysis. Indian Pediatr. 50, 371-376 (2013).

18. Meneses, J., Bhandari, V. \& Alves, J. G. Nasal intermittent positive-pressure ventilation vs nasal continuous positive airway pressure for preterm infants with respiratory distress syndrome: a systematic review and meta-analysis. Arch. Pediatr. Adolesc. Med. 166, 372-376 (2012).

19. Sai Sunil Kishore, M., Dutta, S. \& Kumar, P. Early nasal intermittent positive pressure ventilation versus continuous positive airway pressure for respiratory distress syndrome. Acta Paediatr. 98, 1412-1415 (2009).

20. Kugelman, A. et al. Nasal intermittent mandatory ventilation versus nasal continuous positive airway pressure for respiratory distress syndrome: a randomized, controlled, prospective study. J. Pediatr. 150, 521-526 (2007). 526.e521.

21. Armanian, A. M., Badiee, Z., Heidari, G., Feizi, A. \& Salehimehr, N. Initial treatment of respiratory distress syndrome with nasal intermittent mandatory ventilation versus nasal continuous positive airway pressure: a randomized controlled trial. Int. J. Prev. Med. 5, 1543-1551 (2014).

22. Diblasi, R. M., Zignego, J. C., Smith, C. V., Hansen, T. N. \& Richardson, C. P. Effective gas exchange in paralyzed juvenile rabbits using simple, inexpensive respiratory support devices. Pediatr. Res. 68, 526-530 (2010). 